A-21

\title{
EFECTO DE DIFERENTES CONCENTRACIONES DE NUTRIENTES EN EL FERTIRRIEGO DE UN CULTIVO DE FRESA EN LA PROVINCIA DE HUELVA. EFICIENCIA EN EL USO DEL ABONADO
}

\author{
Molina, $F_{.}{ }^{1}$, Gavilán, P. ${ }^{2}$, Ruiz, ${ }^{2}{ }^{3}$ \\ ${ }^{1}$ Director Departamento Agronómico. Gat Fertiliquidos, Niebla (Huelva). \\ pacomolina@gatfertiliquidos.com \\ ${ }^{2}$ Investigador Titular. IFAPA Centro Alameda del Obispo, Apdo. 3092, 14080 Córdoba, \\ pedrod.gavilan@juntadeandalucia.es \\ ${ }^{3}$ Técnica Especialista Contratada. IFAPA Centro Alameda del Obispo. Apdo. 3092, 14080 \\ Córdoba, natividad.ruiz.baena@juntadeandalucia.es
}

\section{Resumen}

En este trabajo se presentan los resultados de un ensayo de fertirrigación en un cultivo de fresa en la provincia de Huelva. Los objetivos fueron determinar el déficit de fertilizante que se produce al aportar cantidades de riego menores a las normalmente usadas por los agricultores y si se puede compensar con un mayor aporte de fertilizante en la solución de riego, y relacionar la eficiencia en el uso del abonado con la eficiencia en el uso del agua de riego. Para ello se realizó un seguimiento analítico durante toda la campaña y se compararon la solución fertilizante real aportada al cultivo por el sistema de riego con la solución del suelo, extraída con sondas de succión y con muestras de suelo analizadas por el método de extracto saturado. También se realizó un seguimiento y comparación de análisis foliares. No se observaron diferencias importantes ni se produjeron acumulaciones de sales en la zona radicular en tratamientos con distintas concentraciones de abonado en el fertirriego. Las pequeñas diferencias aparecieron en favor de la CE de entrada intermedia, tratamiento T2, que presentó mayores eficiencias y aprovechamiento en el uso de los fertilizantes. Sin embargo, no se apreció esta ventaja en los análisis de hoja ni en la producción.

\section{1- Introducción y Objetivos}

La fresa en gran parte de la provincia de Huelva se cultiva en suelos arenosos, con escaso poder de retención de agua y nutrientes, y se riega con agua que tampoco aporta nutrientes. Se riega con alta frecuencia y, en cierto modo, se puede considerar un cultivo hidropónico en suelo. Por todo ello, la tendencia en la zona es abonar por concentración de nutrientes equilibrados en el agua de riego, es decir utilizan el sistema de manejo proporcional, en el que la cantidad de fertilizantes va ligada a la cantidad de agua.

En el cultivo protegido de la fresa está muy extendido el control de los parámetros climáticos y de humedad del suelo para manejar el riego (Gavilán y col., 2014). Sin embargo, los sistemas de control de la nutrición han tenido menor grado de desarrollo. Se conocen la concentración y equilibrios de sales necesarios para la fertirrigación con volúmenes de agua de riego superiores a los necesarios para cubrir las necesidades de agua del cultivo, pero no tanto para volúmenes de riego más ajustados a las necesidades del cultivo.

Gat Fertiliquidos, empresa fabricante de fertilizantes líquidos, en colaboración con el IFAPA, estudia la fertirrigación de la fresa en la provincia de Huelva, para poder ayudar a los agricultores a fertirrigar de forma más eficiente. 
Al comenzar los ensayos sobre riego, se planteaba la duda de si se produciría un déficit de fertilizante al bajar el volumen de agua de riego, manteniendo las concentraciones de fertilizantes que actualmente se aplican al cultivo con el fertirriego. Es decir, si al recibir menos volumen de riego $\mathrm{y}$, por tanto, menos fertilizante sería necesario compensarlo con un aumento de la concentración del mismo en el agua de riego.

Los objetivos de este trabajo fueron: 1) Determinar si cantidades de riego menores a las normalmente usadas se podrían compensar con un mayor aporte de fertilizante, para mejorar su absorción. 2) Relacionar la eficiencia en el uso del abonado con la eficiencia en el uso del agua de riego.

\section{2- Materiales y Métodos}

Los trabajos se realizaron en una parcela comercial del T.M. de Almonte, en un suelo arenoso, sobre un cultivo de fresa (Fragaria $x$ ananassa) de la variedad Fortuna durante la campaña 2014/15. Se realizó un ensayo con cuatro tratamientos. Los tres primeros tratamientos recibieron volúmenes similares de riego, con el objetivo de cubrir las necesidades de agua del cultivo de forma que T1, T2 y T3 recibieron 4402, 4417, y 4532 $\mathrm{m}^{3} / \mathrm{ha}$, respectivamente. La diferencia entre ellos estuvo en la concentración de fertilizantes aplicada con la fertirrigación. T1 aplicó una solución con una conductividad eléctrica (CE) superior en un $50 \%$ a la de la finca, mientras que T2 y T3 aplicaron conductividades eléctricas un $25 \%$ superior e igual a la de la finca, respectivamente. T4 recibió el volumen de riego tradicional de la finca y la misma CE que T3.

Se trató, por tanto, de compensar los tratamientos con menos agua y fertilizante con un aumento de la CE, y comparar la nutrición mineral de la planta en cada uno de ellos.

Se realizó un seguimiento analítico mensual, desde noviembre hasta mayo, en el que se analizaron parámetros como $\mathrm{CE}, \mathrm{pH}$, bicarbonatos y macronutrientes, expresados en $\mathrm{mS} / \mathrm{cm}$ y $\mathrm{mmol} / \mathrm{l}$, respectivamente. Para determinar el contenido de fertilizante aportado se analizó la solución fertilizante real (SFR) que aportaba el sistema de riego. Para determinar lo que la planta tenía a su disposición se analizó la solución del suelo ( SS $_{\text {sonda }}$ ) a $15 \mathrm{~cm}$ de profundidad, usando sondas de succión, y se tomaron muestras de suelo analizadas por el método de extracto saturado $\left(\mathrm{SS}_{\mathrm{Extr} \text {. Sat. }}\right)$ a $20 \mathrm{~cm}$ de profundidad.

Para comparar la concentración de nutrientes que la planta tenía a su disposición en el suelo (SS) con la concentración que le entraba con el sistema de fertirrigación en cada momento (SFR), se usaron las relaciones "SS sonda $_{\text {ISFR" y "SS }}$ Extr. Sat. $/ S F R$ " de cada ion.

Para la eficiencia en el uso del abonado se convirtieron los nutrientes analizados de unidades de concentración (mmol/litros) a Unidades Fertilizantes ( $\mathrm{kg} / 100$ litros) realmente aplicadas en cada tratamiento por cada 100 litros de agua de riego y se restó las unidades fertilizantes de salida por su volumen de agua de drenaje para calcular su aprovechamiento por la planta. Se expresó en \% de cada volumen de solución, es decir por cada 100 litros de agua de riego que entraba cuantas unidades fertilizantes aportaban, y su correspondiente volumen de drenaje cuantas aportaba. Luego se calculó la relación entre esta eficiencia en el uso del abonado con la del agua de riego.

Por último, se recogieron muestras vegetales tomando hojas adultas representativas de cada tratamiento. Se analizaron todos los macro y microelementos.

\section{3- Resultados}

\subsection{Evolución de los fertilizantes aplicados en cada tratamiento (SFR)}

Los valores de CE de las muestras analizadas durante la campaña de la SFR o entrada al cultivo, fue la indicada en la Tabla 1.

En la finca (tratamiento T4) comenzaron la campaña con valores de 0,55 y llegaron hasta $1 \mathrm{mS} / \mathrm{cm}$ al final del cultivo. 
Tabla 1: Evolución de la CE de la SFR de entrada al cultivo en cada tratamiento $(\mathrm{mS} / \mathrm{cm})$ :

\begin{tabular}{|c|c|c|c|c|c|c|c|}
\hline Gotero & Nov & Dic & Ene & Feb & Mar & Abr & May \\
\hline T1 & 0,78 & 1,15 & 1,45 & 1,36 & 1,48 & 1,44 & 1,48 \\
\hline T2 & 0,66 & 1,00 & 1,15 & 1,19 & 1,20 & 1,12 & 1,24 \\
\hline T3 & 0,58 & 0,80 & 1,02 & 0,92 & 0,96 & 0,98 & 0,89 \\
\hline T4 & 0,55 & 0,80 & 0,81 & 0,92 & 0,96 & 0,98 & 0,89 \\
\hline
\end{tabular}

\subsection{Evolución de los nutrientes en la solución de suelo (SS) en cada tratamiento}

Los valores de CE de las muestras analizadas durante la campaña en la solución de suelo, fue la indicada en la tabla 2 y 3.

La solución de suelo se analizó con dos métodos, utilizando sondas de succión ( SS $_{\text {sonda }}$ ) y tomando muestras de suelo analizadas mediante extracto saturado (SS $\mathrm{Extr}_{\text {. Sat. }}$ ). En la solución del suelo los valores fueron superiores en T1, el tratamiento que recibió menos volumen de agua y mayor CE, mientras que fueron menores en T4, el tratamiento que recibió más volumen de agua y la CE más baja.

Tabla 2: Evolución de la CE de la Solución de suelo de Sonda ( $\mathrm{SS}_{\text {sonda }}$ ) en cada tratamiento (mS/cm):

\begin{tabular}{|c|c|c|c|c|c|c|c|c|}
\hline Sonda & $21 / 11 / 14$ & $23 / 12 / 14$ & $25 / 01 / 15$ & $25 / 02 / 15$ & $25 / 03 / 15$ & $28 / 04 / 15$ & $28 / 05 / 15$ & Promedio \\
\hline T1 & 0,67 & 0,36 & 0,84 & 1,25 & 1,74 & 1,70 & 3,53 & 1,44 \\
\hline T2 & 0,62 & 1,13 & 0,77 & 1,06 & 1,16 & 1,07 & 1,68 & 1,07 \\
\hline T3 & 0,68 & 1,40 & 0,49 & 0,68 & 0,98 & 1,56 & 1,49 & 1,04 \\
\hline T4 & 0,79 & 0,67 & 0,43 & 0,77 & 0,85 & 0,75 & 1,27 & 0,79 \\
\hline
\end{tabular}

Tabla 3: Evolución de la CE de la Solución de suelo de Sonda ( $\mathrm{SS}_{\text {Extr. Sat.) }}$ en cada tratamiento $(\mathrm{mS} / \mathrm{cm})$ :

\begin{tabular}{|c|c|c|c|c|c|c|c|c|}
\hline Suelo & $21 / 11 / 14$ & $23 / 12 / 14$ & $25 / 01 / 15$ & $25 / 02 / 15$ & $25 / 03 / 15$ & $28 / 04 / 15$ & $28 / 05 / 15$ & Promedio \\
\hline T1 & 1,26 & 0,52 & 0,81 & 0,54 & 0,83 & 0,71 & 0,85 & 0,79 \\
\hline T2 & 0,49 & 0,42 & 0,78 & 0,60 & 0,73 & 0,66 & 0,83 & 0,64 \\
\hline T3 & 0,40 & 0,30 & 0,83 & 0,36 & 0,79 & 0,66 & 0,64 & 0,57 \\
\hline T4 & 0,70 & 0,45 & 0,70 & 0,47 & 0,51 & 0,55 & 0,59 & 0,57 \\
\hline
\end{tabular}

\subsection{Comparación de las relaciones solución de nutrientes del Suelo/Entrada al cultivo en cada tratamiento (SS/SFR)}

En cada tratamiento se compararon los valores promedios, de los análisis de toda la campaña, de la solución que quedaba en el suelo con la solución nutritiva que entraba al sistema suelo-planta (Tabla 4 y 5 ).

Presentó menor concentración de nutrientes en la solución del suelo (SS) respecto a la SFR el tratamiento T2, que recibió menor volumen de agua de riego y CE intermedia. $Y$ presentó mayor concentración de nutrientes y mayor valor de esta relación de CE el tratamiento T4, que recibió mayor volumen de riego y una CE similar al T1. Sin embargo estas diferencias fueron pequeñas.

Tabla 4. Comparación de valores promedios de las relaciones $\mathrm{SS}_{\text {Sonda }} / \mathrm{SFR}$. (CE $\mathrm{mS} / \mathrm{cm}$, iones $\mathrm{mmol} \mathrm{I}^{-1}$ )

\begin{tabular}{|c|c|c|c|c|c|c|c|c|c|}
\hline $\begin{array}{c}\text { Sonda } \\
\text { SS/SFR }\end{array}$ & $\mathrm{CE}$ & $\mathrm{NO}_{3}^{-}$ & $\mathrm{PO}_{4} \mathrm{H}_{2}^{-}$ & $\mathrm{SO}_{4}{ }^{=}$ & $\mathrm{Cl}^{-}$ & $\mathrm{Na}^{+}$ & $\mathrm{K}^{+}$ & $\mathrm{Ca}^{++}$ & $\mathrm{Mg}^{++}$ \\
\hline $\mathrm{T} 1$ & 1,06 & 1,06 & 0,67 & 1,84 & 1,57 & 1,76 & 0,38 & 1,40 & 1,73 \\
\hline $\mathrm{T} 2$ & 0,99 & 0,78 & 0,25 & 5,89 & 6,98 & 1,30 & 0,37 & 1,96 & 6,42 \\
\hline
\end{tabular}




\begin{tabular}{|c|c|c|c|c|c|c|c|c|c|}
\hline T3 & 1,20 & 1,02 & 0,72 & 7,00 & 1,87 & 1,61 & 0,40 & 1,72 & 2,11 \\
\hline T4 & 0,96 & 0,94 & 0,47 & 1,56 & 1,32 & 1,36 & 0,44 & 1,12 & 1,64 \\
\hline
\end{tabular}

Tabla 5. Comparación de valores promedios de las relaciones $\mathrm{SS}_{\text {Extr. Sat. }}$ /SFR. (CE mS/cm, iones $\mathrm{mmol} \mathrm{I}^{-1}$ )

\begin{tabular}{|c|c|c|c|c|c|c|c|c|c|}
\hline $\begin{array}{c}\text { Suelo } \\
\text { SS/SFR }\end{array}$ & $\mathrm{CE}$ & $\mathrm{NO}_{3}^{-}$ & $\mathrm{PO}_{4} \mathrm{H}_{2}^{-}$ & $\mathrm{SO}_{4}{ }^{-}$ & $\mathrm{Cl}^{-}$ & $\mathrm{Na}^{+}$ & $\mathrm{K}^{+}$ & $\mathrm{Ca}^{++}$ & $\mathrm{Mg}^{++}$ \\
\hline $\mathrm{T} 1$ & 0,67 & 0,45 & 0,39 & 17,76 & 0,87 & 1,08 & 0,31 & 1,42 & 1,06 \\
\hline $\mathrm{T} 2$ & 0,60 & 0,49 & 0,39 & 8,81 & 0,82 & 1,13 & 0,25 & 0,95 & 0,92 \\
\hline $\mathrm{T} 3$ & 0,64 & 0,46 & 0,41 & 8,22 & 0,74 & 1,11 & 0,22 & 0,96 & 0,84 \\
\hline $\mathrm{T} 4$ & 0,71 & 0,59 & 0,49 & 3,63 & 0,78 & 1,06 & 0,33 & 1,07 & 1,13 \\
\hline
\end{tabular}

\subsection{Comparación de la acumulación de sales}

Para el cálculo de la acumulación de sales en la zona de absorción radicular del cultivo se utilizó la siguiente formula: ((CE $\left.\left.E_{S S}-C E_{S F R}\right) / C E_{S S}\right) \times 100$.

La CE que tenía la solución de suelo (SS) fue menor a la de entrada, en todos los tratamientos y en gran parte de la campaña. Por eso estos valores presentaron valores negativos o muy pequeños, es decir no se produjo acumulación de sales ni $\mathrm{Cl}^{-}$ni de $\mathrm{Na}^{+}$en ningún tratamiento.

Tabla 6. Valores promedios \% drenaje de la CE (CE mS/cm).

\begin{tabular}{|c|c|c|c|c|c|c|c|c|}
\hline Sonda & $21 / 11 / 14$ & $23 / 12 / 14$ & $25 / 01 / 15$ & $25 / 02 / 15$ & $25 / 03 / 15$ & $28 / 04 / 15$ & $28 / 05 / 15$ & Promedio \\
\hline T1 & $-16,42$ & $-219,44$ & $-72,62$ & $-8,80$ & 14,94 & 15,29 & 58,07 & $-32,71$ \\
\hline T2 & $-6,45$ & 11,50 & $-49,35$ & $-12,26$ & $-3,45$ & $-4,67$ & 26,19 & $-5,50$ \\
\hline T3 & 14,71 & 42,86 & $-108,16$ & $-35,29$ & 2,04 & 37,18 & 40,27 & $-0,92$ \\
\hline T4 & 30,38 & $-19,40$ & $-88,37$ & $-19,48$ & $-12,94$ & $-30,67$ & 29,92 & $-15,79$ \\
\hline Ext.Sat. & $21 / 11 / 14$ & $23 / 12 / 14$ & $25 / 01 / 15$ & $25 / 02 / 15$ & $25 / 03 / 15$ & $28 / 04 / 15$ & $28 / 05 / 15$ & Promedio \\
\hline T1 & 38,29 & $-119,89$ & $-78,57$ & $-151,85$ & $-78,96$ & $-102,25$ & $-74,12$ & $-81,05$ \\
\hline T2 & $-35,52$ & $-139,81$ & $-47,44$ & $-100,00$ & $-63,93$ & $-69,44$ & $-50,30$ & $-72,35$ \\
\hline T3 & $-45,00$ & $-166,67$ & $-22,89$ & $-156,98$ & $-21,21$ & $-48,48$ & $-38,41$ & $-71,38$ \\
\hline T4 & 21,20 & $-77,78$ & $-15,06$ & $-96,58$ & $-88,24$ & $-78,18$ & $-51,62$ & $-55,18$ \\
\hline
\end{tabular}

\subsection{Comparación de la eficiencia del abono/eficiencia riego}

Respecto a la eficiencia del abono ofrecieron valores mayores de eficiencia en solución de suelo el T1 (Tabla 7). En cuanto a la relación eficiencia abono/eficacia riego ofrecieron valores mayores de eficiencia en solución de suelo $\mathrm{s}_{\text {sonda }}$ el T1 y de solución suelo $_{\text {Extr. Sat. }}$ mayor el T3. Presentaron mayor aprovechamiento de nutrientes en $\mathrm{K}, \mathrm{P}, \mathrm{y} \mathrm{N}$, menor en Ca y Mg (Tabla 8).

Tabla 7. Valores promedios Eficiencia de abono y de agua (\%).

\begin{tabular}{|c|c|c|c|}
\hline $\begin{array}{c}\text { Resumen } \\
\text { Consumo }\end{array}$ & $\begin{array}{c}\text { Eficiencia } \\
\text { Abonado }\end{array}$ & $\begin{array}{c}\text { Eficiencia } \\
\text { Agua }\end{array}$ & $\begin{array}{c}\text { Eficiencia Abonado/ } \\
\text { Eficiencia Agua }\end{array}$ \\
\hline Sonda T1 & 58,11 & 63,20 & 0,92 \\
\hline Sonda T2 & 41,91 & 62,70 & 0,67 \\
\hline Sonda T3 & 53,17 & 60,30 & 0,88 \\
\hline Ext. Sat. T1 & 78,38 & 63,20 & 1,24 \\
\hline Ext. Sat. T2 & 77,92 & 62,70 & 1,24 \\
\hline Ext. Sat. T3 & 77,09 & 60,30 & 1,28 \\
\hline
\end{tabular}


Tabla 8. Valores promedios Eficiencia de nutrientes (\%).

\begin{tabular}{|c|c|c|c|c|c|}
\hline Consumo & $\mathrm{N}$ & $\mathrm{P}_{2} \mathrm{O}_{5}$ & $\mathrm{~K}_{2} \mathrm{O}$ & $\mathrm{CaO}$ & $\mathrm{MgO}$ \\
\hline Sonda T1 & 55,12 & 73,90 & 81,49 & 42,08 & 37,96 \\
\hline Sonda T2 & 69,22 & 88,04 & 83,24 & 45,68 & $-76,63$ \\
\hline Sonda T3 & 59,47 & 70,93 & 80,36 & 36,43 & 18,67 \\
\hline Promedio & 61,27 & 77,62 & 81,70 & 41,40 & $-6,67$ \\
\hline Ext. Sat. 1 & 85,24 & 83,25 & 88,72 & 65,98 & 68,71 \\
\hline Ext. Sat. 2 & 82,18 & 81,82 & 89,37 & 69,69 & 66,55 \\
\hline Ext. Sat. 3 & 82,85 & 81,20 & 91,33 & 64,59 & 65,48 \\
\hline Promedio & 83,42 & 82,09 & 89,80 & 66,75 & 66,91 \\
\hline
\end{tabular}

\subsection{Comparación de los análisis foliares y producción}

En cuanto a los análisis foliares, no existieron diferencias de valor en concentración de nutrientes en hoja entre tratamientos (Figura 1). Tampoco existieron diferencias en producción entre los tres tratamientos con el mismo volumen de agua de riego y CE diferentes. Los valores fueron iguales a 923,3, 936,2, 934,8 gr/planta para los tratamientos T1, T2, y T3, respectivamente

Figura 1. Análisis foliares (\%).
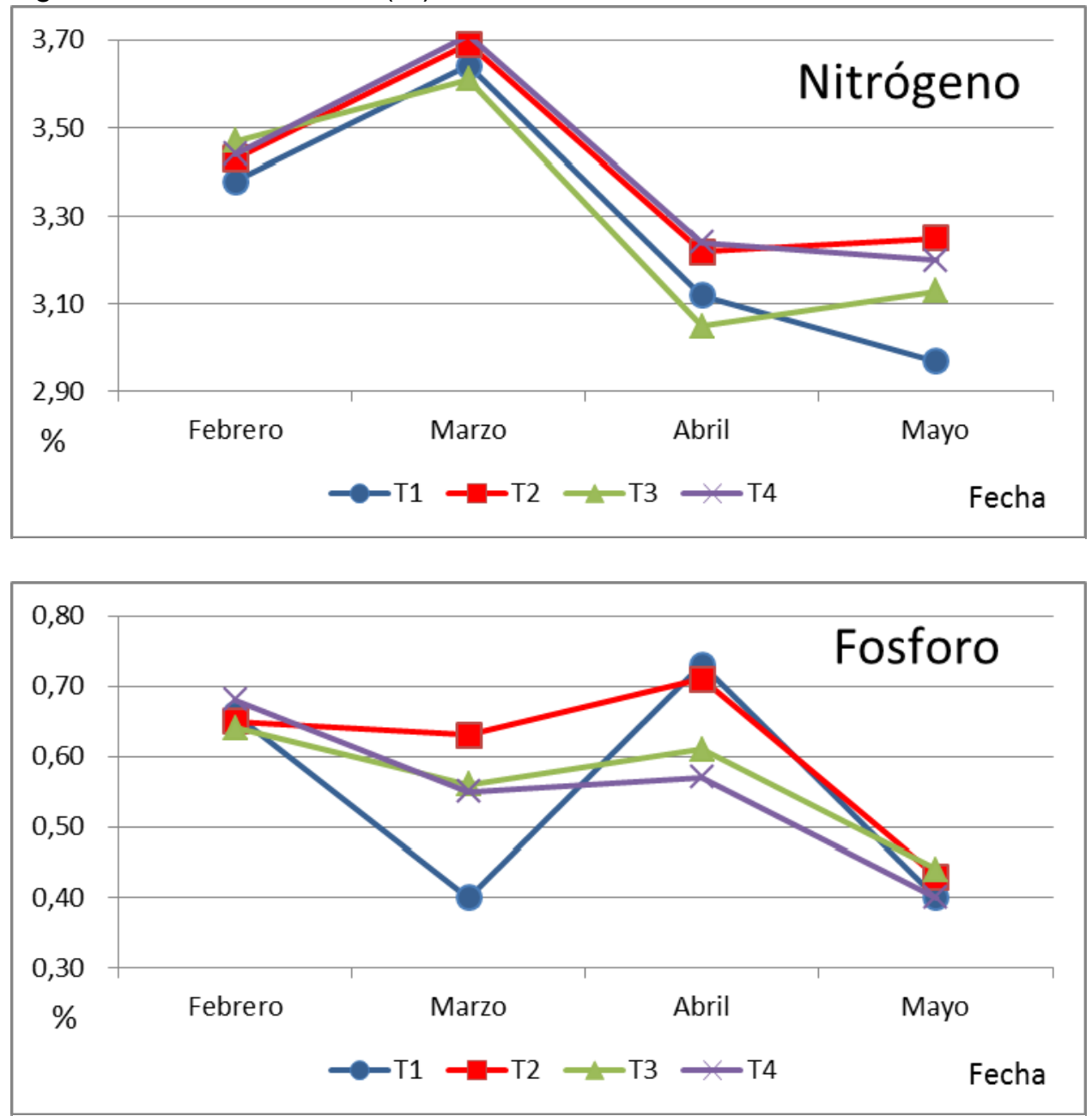

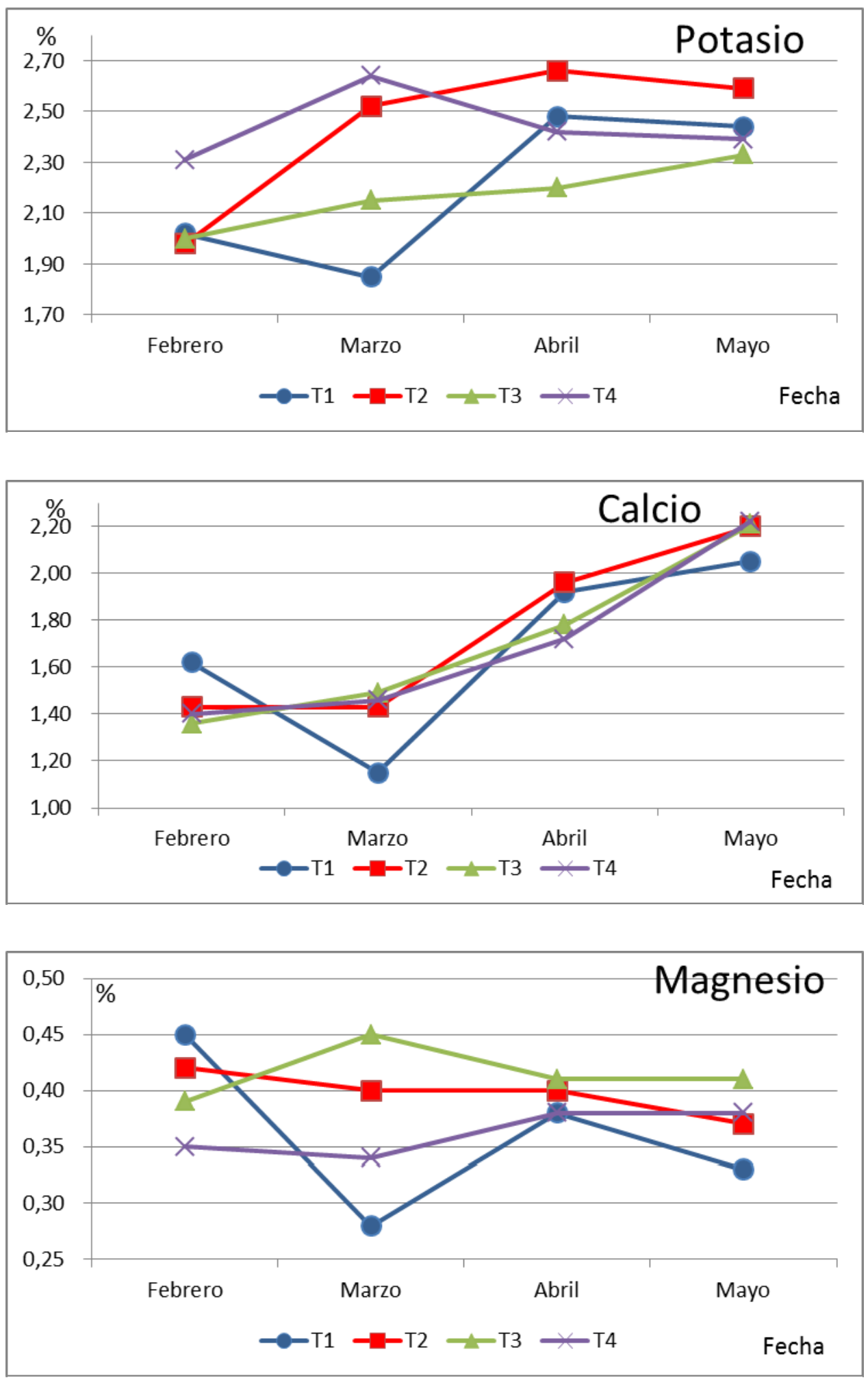

\section{4- Conclusiones y Recomendaciones}

No se observaron grandes diferencias y no se produjeron acumulaciones de sales en zonas radiculares en tratamientos con distintas CE del fertirriego. Las pequeñas diferencias aparecieron en favor de la CE de entrada intermedia, T2, que presentó mayores eficiencias 
y aprovechamiento en el uso de los fertilizantes. Sin embargo, no se aprecia esta ventaja en los análisis de hoja ni en la producción.

Es recomendable aumentar CE en los primeros meses, noviembre a marzo, con volúmenes de riego menores, en los que todos los tratamientos se han quedado bajos de concentración de nutrientes. Y en los siguientes bajarlos, con volúmenes de riego mayores. Así mismo aumentar en los primeros meses la concentración de $\mathrm{N}, \mathrm{P}$, y Ca y luego aumentar $\mathrm{K}$ y $\mathrm{Mg}$. Sería interesante conocer la respuesta del cultivo a estos cambios también en calidad.

\section{Agradecimientos}

Este trabajo no habría podido ser realizado sin el patrocinio de las empresas FRUTAS BORJA y GAT FERTILIQUIDOS.

\section{5- Referencias}

Duchein, M.C., Baile, M., Balle, A., (1994)."Water use efficiency and nutrient consumption of greenhouse rose grown in rockwool". Acta Horticulturae.

Gavilán, P., Lozano, D., Ruiz, N., Molina, F., 2014. El riego de la fresa en el entorno de Doñana. Evapotranspiración, coeficientes de cultivo y eficiencia del riego. XXXII Congreso Nacional de Riegos. Asociación Española de Riegos y Drenajes. Madrid, 1012 junio de 2014.

Cadahía, C. (1988). Fertilización en riego por goteo de cultivos hortícolas, Edit. ERT Fertilizantes, Unión Explosivos Rio Tinto, Madrid. 